\title{
Properties of the spatial Wigner function of entangled photon pairs
}

\author{
Noelia Gonzalez, ${ }^{1, *}$ Gabriel Molina-Terriza, ${ }^{1,2}$ and Juan P. Torres ${ }^{1,3}$ \\ ${ }^{1}$ ICFO-Institut de Ciencies Fotoniques, Mediterranean Technology Park, Castelldefels, 08860 Barcelona, Spain \\ ${ }^{2}$ ICREA-Institució Catalana de Recerca i Estudis Avançats, 08010 Barcelona, Spain \\ ${ }^{3}$ Departament of Signal Theory and Communications, Universitat Politecnica de Catalunya, 08034 Barcelona, Spain
}

(Received 18 May 2009; published 5 October 2009)

\begin{abstract}
In this paper we analyze some important characteristics of the spatial Wigner function of entangled photon pairs. We show that the Wigner description of quantum states that live in the infinite-dimensional spacemomentum degree of freedom proves to be particularly useful. We propose an experimental configuration that can be used to retrieve the Wigner function of paired photons entangled in the spatial degree of freedom. In particular, it allows the full characterization of the paired photons emitted from a spontaneous parametric down-conversion source.
\end{abstract}

DOI: 10.1103/PhysRevA.80.043804

PACS number(s): 42.50.Dv

\section{INTRODUCTION}

The efficient and reliable generation, control and detection of specific states of quantum systems is at the roots of the field of quantum information [1]. One important property of quantum systems composed of several parties is that they can become entangled, i.e., the quantum state of the whole system cannot be described by a set of separable quantum states for each party of the ensemble. Entangled states have found many applications in quantum computing, quantum teleportation, and are at the core of certain cryptographic protocols [1-3].

Quantum systems which present entanglement in continuous variables have been receiving increasing attention from the scientific community due to their potentiality. The most widely explored continuous variable system is based on the quadratures of the electrical field of photons. The transverse spatial shape of photons (i.e., transverse position and momentum degree of freedom) is another of such continuous variable systems. It has already been shown that pairs of photons can be entangled in their spatial properties [4]. Up to now, the entanglement in transverse momentum has been used to test some quantum protocols in a finite number of dimensions [5-8]. On the other hand, due to its ease of control, the spatial degree of photons is prone to be used for testing some of the new physical predictions that continuous variables quantum systems can offer [9].

One suitable representation of the quantum state of a system is the Wigner function [10,11]. The Wigner-function formulism is fully equivalent to the density matrix representation, thus providing all the accessible information of the system to the observer. Wigner functions are especially useful for describing continuous variables. In particular, it has been used for describing the quadratures of the electrical field with coherent and squeezed states [12] or single photon states [13].

Here we analyze the structure of the phase space of entangled photons in the transverse momentum degree of freedom using the Wigner-function formulism. The description

*noelia.gonzalez@icfo.es of the spatial transverse modes of an optical field in terms of the Wigner function can be found in $[14,15]$. In this paper we present some results which can be directly applied to describe the transverse spatial shape of bipartite entangled photons generated in spontaneous parametric down-conversion (SPDC) processes. Thus, in Sec. II we revise the essential properties of the Wigner function that we will use along the paper and in Sec. III we describe the typical quantum state of the momentum of photons generated in a SPDC process. For the sake of clarity, we analyze the spatial Wigner function in three different situations: First, we consider the case where one of the photons is projected onto a specific pure state, obtaining the Wigner function of the remaining photon (Sec. IV). We also consider the case when one simply disregard one of the photons, the other being, in principle, in a mixed state (Sec. V). Finally we propose an experimental scheme for measuring the spatial properties of the whole entangled state, and analyze the properties of the Wigner function of the whole system (Sec. VI).

\section{WIGNER FUNCTION}

The Wigner function associated with a quantum state $|\Psi\rangle$ can be expressed as the expectation value of the operator $\hat{\Pi}_{\mathbf{r q}}$ [16],

$$
W(\mathbf{r}, \mathbf{q})=\frac{1}{\pi^{2}}\left\langle\Psi\left|\hat{\Pi}_{\mathbf{r q}}\right| \Psi\right\rangle
$$

where $\hat{\Pi}_{\mathbf{r q}}$ is defined as

$$
\begin{aligned}
\hat{\Pi}_{\mathbf{r q}} & =\int d \mathbf{r}_{0} \exp \left(-2 i \mathbf{q} \cdot \mathbf{r}_{0}\right)\left|\mathbf{r}-\mathbf{r}_{0}\right\rangle\left\langle\mathbf{r}+\mathbf{r}_{0}\right| \\
& =\int d \mathbf{q}_{0} \exp \left(-2 i \mathbf{r} \cdot \mathbf{q}_{0}\right)\left|\mathbf{q}+\mathbf{q}_{0}\right\rangle\left\langle\mathbf{q}-\mathbf{q}_{0}\right| .
\end{aligned}
$$

The operator $\hat{\Pi}_{\text {rq }}$ performs a reflection about the phase-space point $(\mathbf{r}, \mathbf{q})$ and is thus the parity operator about that point. In the case of a "two particle" state the Wigner function can be expressed as [17] 


$$
W\left(\mathbf{r}_{1}, \mathbf{q}_{1}, \mathbf{r}_{2}, \mathbf{q}_{2}\right)=\frac{1}{\pi^{4}}\left\langle\Psi_{1,2}\left|\hat{\Pi}_{1,2}\right| \Psi_{1,2}\right\rangle,
$$

where $\hat{\Pi}_{1,2}$ is the product of the two displaced operators, each one acting over one particle,

$$
\hat{\Pi}_{1,2}=\hat{\Pi}_{\mathbf{r}_{1}, \mathbf{q}_{1}} \otimes \hat{\Pi}_{\mathbf{r}_{2}, \mathbf{q}_{2}} .
$$

The Wigner function corresponding to a generally mixed state with density matrix $\rho=\Sigma \lambda_{i}\left|\Psi_{i}\right\rangle\left\langle\Psi_{i}\right|$ can be written, making use of Eq. (3), as $W(\mathbf{r}, \mathbf{q})=\operatorname{Tr}\left(\rho \hat{\Pi}_{\mathbf{r q}}\right)$. An interesting property of the Wigner function, easily obtained from Eqs. (1) and (2), is that the Wigner function of a probabilistic mixtures of density matrices, i.e., $\rho=\Sigma \lambda_{i} \rho_{i}$ is $W=\sum \lambda_{i} W_{i}$. Notwithstanding, this is not the case for a linear superposition of pure states, whose Wigner function does not result in a linear superposition of the corresponding Wigner functions.

\section{SOURCE OF SPATIALLY ENTANGLED PHOTONS}

In the following we will consider the important case of a two-photon state, whose quantum state can be written as

$$
|\Psi\rangle=\int d \mathbf{q}_{s} d \mathbf{q}_{i} \Psi\left(\mathbf{q}_{s}, \mathbf{q}_{i}\right) a_{s}^{\dagger}\left(\mathbf{q}_{s}\right) a_{i}^{\dagger}\left(\mathbf{q}_{i}\right)|0\rangle_{s}|0\rangle_{i},
$$

where $\mathbf{q}_{s}=\left(q_{s, x}, q_{s, y}\right)$ and $\mathbf{q}_{i}=\left(q_{i, x}, q_{i, y}\right)$ are the corresponding transverse momenta of the signal and idler photons, respectively. $a_{s}^{\dagger}\left(\mathbf{q}_{s}\right)$ is the creation operator of a signal photon with transverse momentum $\mathbf{q}_{s}$, and similarly for the idler photon.

In the rest of the paper we will confine ourselves to the case where the probability amplitude $\Psi$ writes

$$
\Psi\left(\mathbf{q}_{s}, \mathbf{q}_{i}\right)=\frac{|A B|^{1 / 2}}{\pi} \exp \left(-A\left|\mathbf{q}_{s}+\mathbf{q}_{i}\right|^{2}-B\left|\mathbf{q}_{s}-\mathbf{q}_{i}\right|^{2}\right),
$$

where $A$ and $B$ are two possibly complex constants that will allow us to analyze different types of momentum correlations among the photons. The quantum state given by Eq. (6) is normalized, so that $\int d \mathbf{q}_{s}, d \mathbf{q}_{i}\left|\Psi\left(\mathbf{q}_{s}, \mathbf{q}_{i}\right)\right|^{2}=1$. The type of states described by Eq. (6) is ubiquitous when describing quantum systems of continuous variables. In the case of momentum correlated photons, the state given by Eq. (6) is a very good approximation for describing paired photons entangled in the momentum degree of freedom $[18,19]$.

In particular, this kind of states can be produced when a second-order nonlinear crystal is illuminated by a quasimonochromatic pump beam in a Gaussian mode in order to produce frequency downconverted waves. The downconverted waves should be generated in a collinear configuration (all interacting waves propagate along the same direction), and the Poynting vector walkoff should be negligible. One way of achieving such conditions is the use of noncritical type-II quasi-phase-matched nonlinear crystals. If such a crystal geometry is chosen, and under the approximation that the refractive indices of pump, signal and idler are nearly equal, Eq. (6) is a good approximation to the state of the photons at the output face of the crystal. The values obtained of the two constants, $A$ and $B$, are [19]

$$
\begin{gathered}
A=\frac{1}{4}\left(\frac{w_{p}^{2}}{1+w_{p}^{4} / \sigma_{0}^{2}}\right)+i \frac{1}{4}\left(\frac{L}{k_{p}^{0}}-\frac{\sigma_{0}}{1+\sigma_{0}^{2} / w_{p}^{4}}\right), \\
B=\frac{\alpha L}{4 k_{p}^{0}}+i \frac{L}{4 k_{p}^{0}},
\end{gathered}
$$

where $L$ is the length of the nonlinear crystal, $\alpha$ is a fitting constant to approximate the phase matching functions sinc function by a Gaussian function (in our case we use $\alpha$ $=0.455), w_{p}$ and $\sigma_{0}$ are the pump beam width and radius of curvature considered at the center of the crystal, respectively, $k_{p}^{0}=\omega_{p} n_{p} / c$, and $\omega_{p}$ and $n_{p}$ are the corresponding angular frequency and refractive index.

From Eq. (6) it can be readily checked that the momentum correlations in each of the two transverse coordinates are completely independent, i.e., there is no cross-correlation between direction $x$ and direction $y$. Therefore, in the rest of the paper we will drop all vector quantities, and focus on just one of the transverse dimensions of the photons.

\section{REMOTE PREPARATION OF A PURE STATE}

The two-photon state described by Eq. (6) is pure. After projecting the idler photon in a pure state, the signal photon will also remain in a pure state. The specific spatial shape of the signal photon will depend on (a) the two-photon momentum correlations of signal and idler and (b) the spatial shape of the mode onto which the idler photon is projected. In this sense, we talk about remote preparation of pure states. Theoretically, whenever Eq. (6) represents a nonseparable state, one could achieve any remote preparation of a given spatial state. This is due to the fact that the two-photon state admits a Schmidt decomposition onto an infinite series of spatial modes, each one of them with a finite, but ever decreasing amplitude. However, in any experimental implementation the limits of control would be given by the precision in the preparation of states, the noise in the generation of the photons and the fundamental limits given by the paraxial approximation.

After projecting the idler photon, the Wigner function of the signal photon can be expressed as

$$
W_{\Phi, s}\left(x_{s}, q_{s}\right)=\frac{1}{\pi^{2}}\left\langle\Psi_{s, i}\left|P_{\Phi, i} \otimes \hat{\Pi}_{x_{s}, q_{s}}\right| \Psi_{s, i}\right\rangle,
$$

where $P_{\Phi, i}=|\Phi\rangle\left\langle\left.\Phi\right|_{i}\right.$ projects the idler photon onto the state $|\Phi\rangle=\int d q \Phi(q) a_{i}^{\dagger}(q)|0\rangle_{i}$. We will exemplify this remote preparation procedure with two different cases: the projection onto a Gaussian mode, and projections onto coherent superpositions of Gaussian and Hermite-Gaussian (HG) modes.

\section{A. Projection onto a Gaussian state}

We describe the projection of the idler photon into a Gaussian state by the projector $P_{G}=|G\rangle\langle G|$ where $|G\rangle$, in the transverse momentum space, is given by

$$
|G\rangle=N_{G} \int d q \exp \left(-\frac{1}{4} \frac{q^{2}}{\mu_{x}}\right)|q\rangle,
$$

$N_{G}$ is a normalization constant and $\mu_{x}$ is the complex beam width in real space given by 

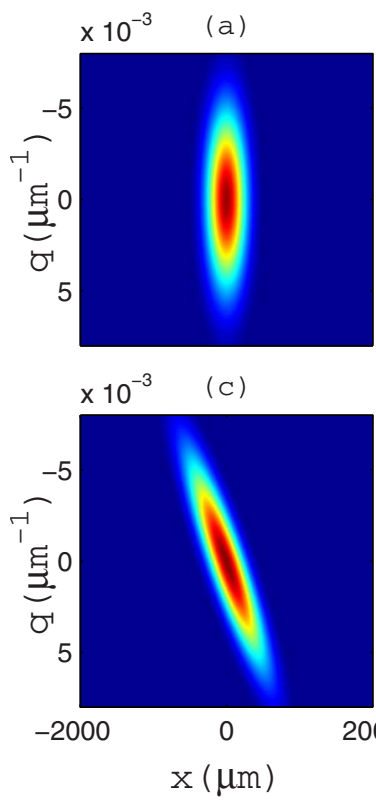

(b)

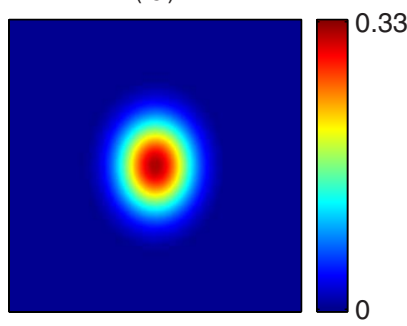

(d)

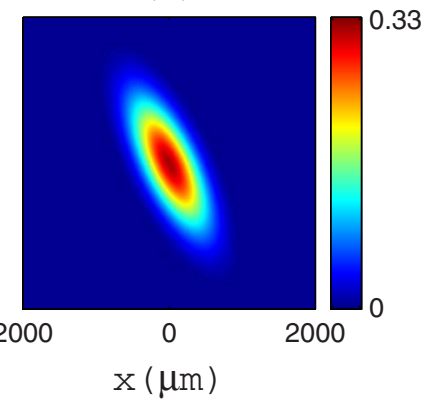

FIG. 1. (Color online) Examples of remote preparation of a single photon in a spatial Gaussian state. The pump is a Gaussian mode, and the idler is projected onto a Gaussian state. (a) and (b) show two Wigner functions with no curvature of the idler mode $\left(R_{i} \rightarrow \infty\right)$, and different values of the beam width of the idler photon $w_{i}$ : (a) $w_{i}=0.4 \mathrm{~mm}$, (b) $w_{i}=1 \mathrm{~mm}$. (c) and (d) correspond to the same beam width of idler photon, $w_{i}=1 \mathrm{~mm}$, but different values of the radius of curvature, (c) $R_{i}=1 \mathrm{~m}$, (d) $R_{i}=200 \mathrm{~m}$. In all cases: beam width of the pump beam $w_{p}=1 \mathrm{~mm}$; no curvature of the pump beam $\left(R_{p} \rightarrow \infty\right)$; Length of the nonlinear crystal $L=5 \mathrm{~mm}$.

$$
\mu_{x}=\frac{1}{w_{0}^{2}}+i \frac{k}{2 R} .
$$

$w_{0}$ is the beam waist, $k$ is the longitudinal idler wave vector and $R$ is the radius of curvature of the beam. Making use of Eq. (8), we find that the Wigner function for the signal photon reads

$$
W_{G, s}\left(x_{s}, q_{s}\right)=\frac{1}{\pi} \exp \left(-h_{R} q_{s}^{2}\right) \exp \left(-\frac{\left(x_{s}-h_{I} q_{s}\right)^{2}}{h_{R}}\right),
$$

where $h_{G}=\left(h_{R}+i h_{I}\right) / 2$ is defined as

$$
h_{G}=(A+B)-\frac{(B-A)^{2}}{B+A+1 /\left(4 \mu_{x}^{*}\right)} .
$$

Since the biphoton function given by Eq. (6) describes perfect correlations in orbital angular momentum (OAM) between the signal and idler photons $\left[m_{s}+m_{i}=0\right.$, with $m_{s, i}$ being the OAM index of the signal (idler) beams] [20], Eq. (11) correspond to the Wigner function of a pure Gaussian state.

In Fig. 1 we show a few examples of the remote preparation of Gaussian states as given by Eq. (11) and (12). Note the different features of the Wigner function. For example, tilting of the Wigner function appears whenever the state presents curvature, i.e., when either $A, B$, or $\mu_{x}$ have nonvanishing imaginary parts. In Figs. 1(a) and 1(b) the Wigner function it is not tilted because the chosen parameters make $A, B$, and $\mu_{x}$ purely real ( $L$ small and $R_{i}$ and $R_{p}$ tending to
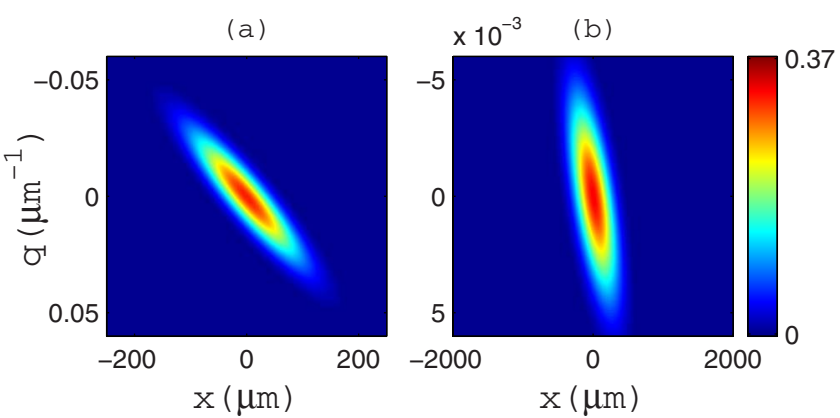

FIG. 2. (Color online) Effect of the pump beam on the remote preparation of a Gaussian state (a)no curvature of the pump beam $\left(R_{p} \rightarrow \infty\right), w p=38.23 \mu \mathrm{m}$ and $L=50 \mathrm{~mm}$ satisfying $A=B ;(\mathrm{b})$ $R_{p}=2 \mathrm{~m}, w_{p}=1 \mathrm{~mm}$, no curvature of the idler mode $\left(R_{i} \rightarrow \infty\right)$, $w_{i}=0.5 \mathrm{~mm}, L=5 \mathrm{~mm}$.

infinitive). Also, note that the two transverse widths of the elliptical Wigner function, in the $q_{s}$ and $\left(x_{s}-h_{I} q_{s}\right)$ directions, are inversely related as shown in Eq. (11). We will see below that this only happens when the represented state is pure and can be clearly observed by comparing Figs. 1(a) and 1(b).

Also, the idler Gaussian width only enters Eq. (11) through $h_{G}$. From Eq. (12) it is readily observed that when $A=B$, the spatial shape of the signal photon is independent of the coincident idler projection. This could have already been anticipated since Eq. (6) represents a separable state whenever $A=B$ [19]. In Fig. 2 we plot two cases with different pumps but fixed idler projections. In particular, Fig. 2(a) shows the especial case when $A=B$.

\section{B. Projection onto superpositions of Hermite-Gaussian and Gaussian states}

Now we consider a multimode situation, i.e., the idler photon is projected onto a coherent superposition of two modes: a first order Hermite-Gaussian (HG) mode and a Gaussian mode. The idler photon projector into a coherent superposition can be written as $P_{\mathrm{HG}}=|\mathrm{HG}\rangle\langle\mathrm{HG}|$, where

$$
|\mathrm{HG}\rangle=N_{\mathrm{HG}} \int d q\left(2 q_{1}+i q\right) \exp \left(-\frac{1}{4} \frac{q^{2}}{\mu_{x}}\right)|q\rangle,
$$

where $q_{1}=x_{1} \mu_{x}$ gives the relative amplitude between the pure Gaussian and the pure Hermite-Gaussian modes being $x_{1}$ the position of the null appearing in the field in real space. When $\mu_{x}$ is purely real, then $q_{1}=x_{1} / w_{0}^{2}$. The Wigner function corresponding to the quantum state of the signal, after projecting the idler onto the mode given by Eq. (13) reads

$$
W_{\mathrm{HG}, s}\left(x_{s}, q_{s}\right)=N_{\mathrm{HG}} C_{H, s}\left(x_{s}, q_{s}\right) W_{G, s}\left(x_{s}, q_{s}\right),
$$

where

$$
\begin{aligned}
C_{H, s}\left(x_{s}, q_{s}\right)= & \left|g_{0}\right|^{2} q_{s}^{2}+\frac{\left|g_{0}\right|^{2}}{h_{R}^{2}}\left(h_{I} q_{s}+x_{s}\right)^{2}-\frac{\left|g_{0}\right|^{2}}{2 h_{R}}-4 \operatorname{Im}\left(q_{1}^{*} g_{0}\right) q_{s} \\
& -4 \frac{\operatorname{Re}\left(q_{1}^{*} g_{0}\right)}{h_{R}}\left(h_{I} q_{s}+x_{s}\right)+4\left|q_{1}\right|^{2},
\end{aligned}
$$

$W_{G, s}$ is defined by Eq. (11) and $g_{0}$ writes 


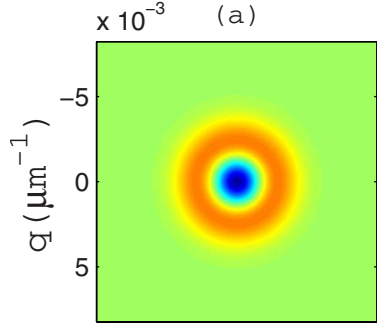

$\times 10^{-3} \quad(\mathrm{C})$

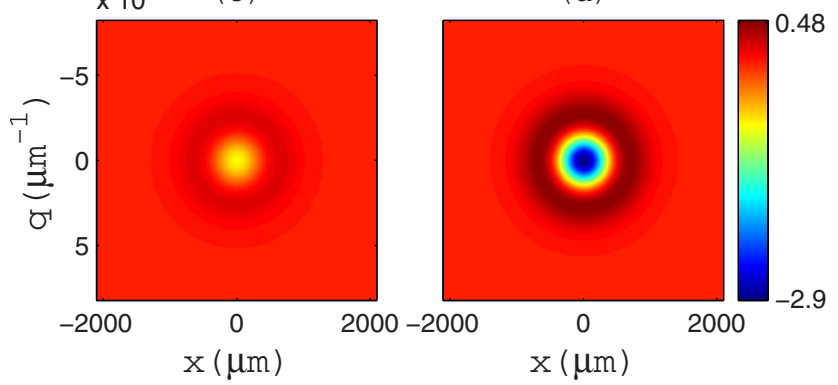

FIG. 3. (Color online) Examples of remote preparation of a single photon in a spatial superposition of a Gaussian and a Hermite Gaussian state for different values of $q_{1}$. The pump is a Gaussian mode, whereas the idler photon is projected onto a given superposition of the states. (a) and (b) show two cases where $q_{1}$ is purely real, whereas in (c) and (d) $q_{1}$ is purely imaginary. (a) $q_{1}=0$, (b) $q_{1}=2 \times 10^{-4} \mu \mathrm{m}$, (c) $q_{1}=i 10^{-8} \mu \mathrm{m}$, (d) $q_{1}=i 2 \times 10^{-8} \mu \mathrm{m}$. In all cases: Beam width of the idler and pump: $w_{i}=w_{p}=1 \mathrm{~mm}$; curvature of the idler photon and the pump beam $R_{i}=R_{p}=100 \mathrm{~m}$; Crystal length $L=5 \mathrm{~mm}$.

$$
g_{0}=\frac{B-A}{B+A+1 /\left(4 \mu_{x}^{*}\right)} .
$$

Notice again that when $A=B, g_{0}=0$ and two-photon state is separable. In this situation the Wigner function of the signal photon corresponds to a Gaussian one. When the two photons are in a separable state, if we set $q_{1}=0$, i.e., we project the idler into a pure Hermite-Gaussian mode, the probability for a signal-idler coincidence is exactly zero [20]. Some examples of the shapes described by Eq. (14) are shown in Fig. 3 . Note that the superposition of a Gaussian and a HermiteGaussian state does not result on the sum of their respective Wigner functions.

\section{WIGNER FUNCTION OF THE SIGNAL PHOTON AND THE AMOUNT OF SPATIAL ENTANGLEMENT}

In this section we consider the spatial properties of the signal photon alone, i.e., regardless of the state of the idler photon. The Wigner function of the signal photon alone can be calculated from the two-photon state given by Eq. (6) using

$$
W\left(x_{s}, q_{s}\right)=\frac{1}{\pi^{2}} \operatorname{Tr}\left[\left\langle\Psi_{s, i}\left|\hat{I}_{i} \otimes \hat{\Pi}_{x_{s}, q_{s}}\right| \Psi_{s, i}\right\rangle\right],
$$

which corresponds to calculating the Wigner function of the signal photon alone, applying the identity and tracing out the idler photon. The Wigner function obtained reads

$$
\begin{aligned}
W_{s}\left(x_{s}, q_{s}\right)= & \frac{1}{\pi} \sqrt{\frac{H_{R}-G}{H_{R}+G}} \exp \left\{-2\left(H_{R}-G\right) q_{s}^{2}\right\} \\
& \times \exp \left\{\frac{-\left[x_{s}-2 H_{I} q_{s}\right]^{2}}{2\left(H_{R}+G\right)}\right\}
\end{aligned}
$$

where $H$ and $G$ are defined as

$$
\begin{gathered}
H=H_{R}+i H_{I}=(A+B)-\frac{(A-B)^{2}}{2 \operatorname{Re}(A+B)}, \\
G=\frac{|A-B|^{2}}{2 \operatorname{Re}(A+B)} .
\end{gathered}
$$

Note that in this case, the purity of the quantum state that describes the signal photon, which can be easily calculated once the Wigner function has been measured, can be used to determine the degree of entanglement of the initial twophoton state. The purity of the signal photon can be calculated as $P=P_{x}^{2}$, where $P_{x}=2 \pi \int d x_{s} d q_{s} W_{s}\left(x_{s}, q_{s}\right)^{2}$ and reads

$$
P_{x}=\left(\frac{H_{R}-G}{H_{R}+G}\right)^{1 / 2} .
$$

From Eqs. (18) and (21) it can be seen than the purity of the signal photon is directly related to the product of the two transverse widths of the elliptical Wigner function, in the $q_{s}$ and $\left(x_{s}-2 H_{I} q_{s}\right)$ directions, respectively, satisfying the constrain

$$
P_{x}=\Delta_{q_{s}} \Delta_{\left(x_{s}-2 H_{I} q_{s}\right)} \leq 1 .
$$

Some comments are in order: first, notice again that if $A$ $=B, G=0$ and the two-photon state is separable. In this case the Wigner function of the signal photon corresponds to the Wigner function of a Gaussian state with the same conditions, so that the state of the signal photon is pure. Note that in this situation the two transverse widths of the Wigner function are inversely related and as it is expected the purity of the signal estate given by Eq. (22) is $P_{x}=1$. Finally, we can quantify the degree of entanglement of the initial state with the von Neumann entropy $S_{x}$, which in this case depends only with the purity of the traced state in the following way $[21,22]$ :

$$
S_{x}\left(P_{x}\right)=\frac{1-P_{x}}{2 P_{x}} \ln \left(\frac{1+P_{x}}{1-P_{x}}\right)-\ln \left(\frac{2 P_{x}}{1+P_{x}}\right) .
$$

Some numerical examples of the Wigner function given by Eq. (18) are shown in Figs. 4(a) and 4(c). For Fig. 4(c) we have chosen $w_{p}$ and $L$ to satisfy the condition $A=B$. As it is expected, in this case the two-photon state, which is separable, gives the Wigner function of a Gaussian state with equal conditions. It is clear if we compare Fig. 4(c) with Fig. 2(a) which shows the same Wigner function. Also, note that comparing Fig. 4(a) with Fig. 1(b) it is clearly seen how the purity of the former case is less than one. 
(a)

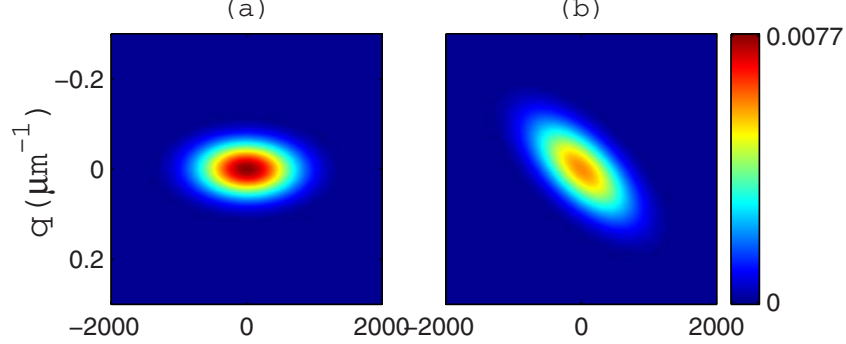

(c)

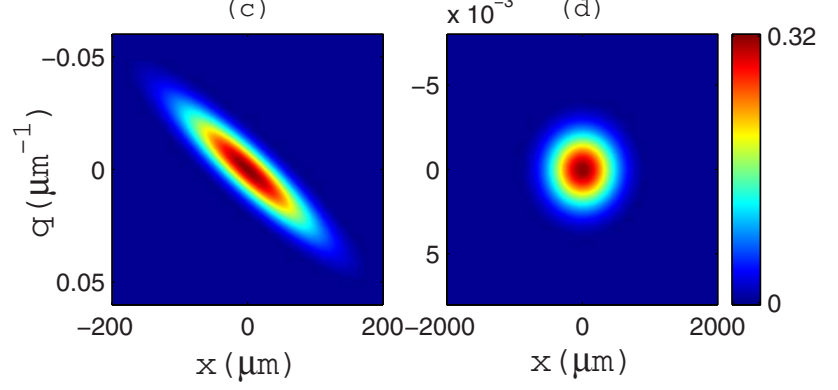

FIG. 4. (Color online) Examples of the Wigner function of the single photon alone, i.e., without considering the idler photon. The pump is a Gaussian beam. (a) and (c) show two cases where the signal photon is analyzed at the output face of the nonlinear crystal. In (b) and (d) we have considered the propagation of the signal photon using a lens with focal length $f \mathrm{~mm}$ located $L_{1}$ after the output face of the nonlinear crystal, and at $L_{2}$ from the entrance of the interferometer. For (a) and (b) $w_{p}=1 \mathrm{~mm}$ and $L=5 \mathrm{~mm}$. For (c) and (d) $w_{p}=38.23 \mu \mathrm{m}$ and $L=50 \mathrm{~mm}$ (satisfying the condition $A$ $=B$. In (b) $f=150 \mathrm{~mm}, L_{1}=300 \mathrm{~mm}$ and $L_{2}=300 \mathrm{~mm}$, in (d) $f$ $=200 \mathrm{~mm}, L_{1}=175 \mathrm{~mm}$ and $L_{2}=200 \mathrm{~mm}$. No curvature of the pump beam: $R_{p} \rightarrow \infty$ for all cases.

\section{EXPERIMENTAL PROPOSALS FOR MEASURING THE WIGNER FUNCTION}

\section{A. Measurement of the Wigner function of the signal photon}

The Wigner function of the signal photon described in the previous sections can be measured experimentally using an extension of the method proposed in [23]. Figure 5 shows the scheme proposed to measure the Wigner function of the signal from the entangled two-photon pair. The Wigner function of the signal photon is measured by means of a threedimensional (3D) Sagnac interferometer. The detailed description of this device can be found in [23]. At the output of the 3D Sagnac interferometer, the two beams which counterpropagate, produce an interference pattern which is then sent to a detector. The role of the out of the plane reflections (round mirror in the figure) is to spatially invert one of the interfering beams with respect to the other, in order to produce the parity operation described in Eq. (2). On the other hand, the input beam is properly displaced and tilted to complete the operator in Eq. (2). Under these experimental conditions, the coincidences rates from the both output ports, signal and idler, will have the following shape:

$$
R_{c} \propto\left[I_{s}^{1}+I_{s}^{2}+2 \gamma \tilde{W}_{s}\left(x_{s}, q_{s}\right)\right] I_{i}
$$

where $I_{i}$ is the detected idler photon flux, $I_{s}^{n}=\alpha_{n} I_{s}$, with $n$ $=1,2$, are proportional to the counts corresponding to the

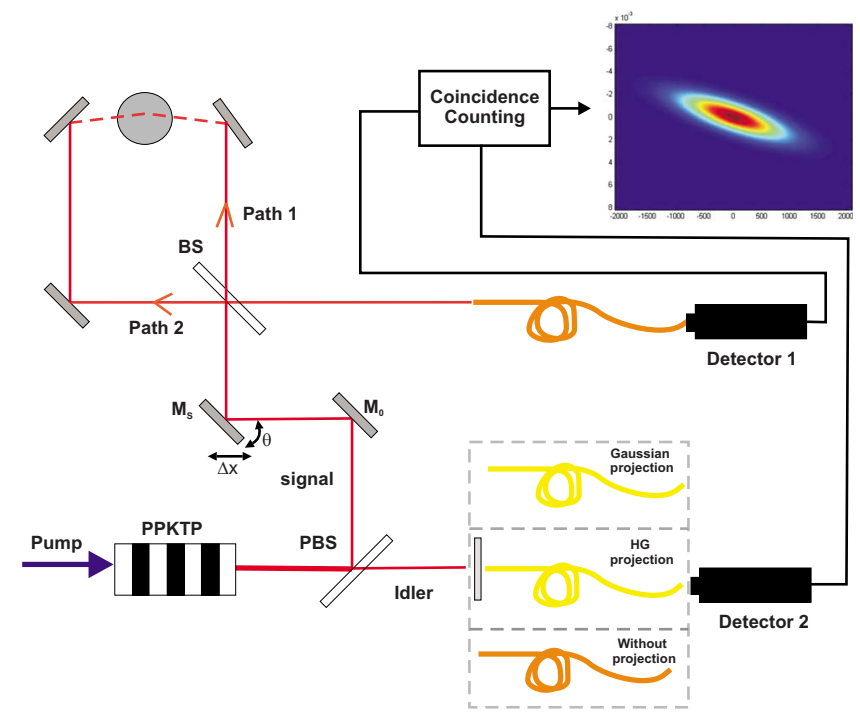

FIG. 5. (Color online) Sketch of the setup proposed for the retrieval of the Wigner function of a remotely prepared photon. Pairs of momentum entangled photons are generated from a nonlinear crystal illuminated by a quasimonochromatic Gaussian pump beam in a collinear configuration. The signal and idler photon follow different paths after traversing a PBS. The idler photon is detected by Detector2 after been collected using the appropriate optical elements depending on the remote preparation that we want to perform. The signal photon is detected by Detector 1 after passing through a Sagnac interferometer, and collected with a multimode fiber. Using coincident logical detection, we measure the Wigner function of the signal photon.

two counterpropagating directions of the signal photon in the interferometer. Here, $I_{s}$ is the photon flux rate of the incoming signal beam and $\alpha_{1}=R^{2}\left(\alpha_{2}=T^{2}\right)$ depends on the reflectivity, $R$ (transmissivity $T$ ) of the beam-splitter. On the other hand, $\gamma=R T$ and $\tilde{W}_{s}\left(x_{s}, q_{s}\right)$ is proportional to the Wigner function of the signal photon. Therefore, the proper Wigner function can be extracted from the measurement of the coincidences of the two outputs, after subtracting the background and properly normalizing the result.

First, let us describe the basic setup for measuring the quantum state of the signal once the idler has been projected onto a Gaussian state (Sec. IV A). In this case, we collect the idler photon with a single mode fiber (SMF) and detect it, thus effectively projecting the idler onto a Gaussian state. We record the coincident signal photons after it has traversed the interferometer. Finally, after subtracting the coincidences background, we obtain an interference pattern which is proportional to the Wigner function, as given by Eq. (11). Note that the value of $\mu_{x}$ in Eq. (9) is the one of the idler beam at the output face of the crystal, thus one has to take into account the propagation from the fiber to the crystal.

The projection in a superposition of Gaussian and Hermite-Gaussian states [Sec. IV B] can be done in a similar way, but now introducing a mode transformation of the idler spatial profile before the projection operation implemented by the SMF. The mode transformation together with the projection onto a Gaussian state can be seen as a projection onto a HG state. Again, the proper values of $q_{1}$ and $\mu_{x}$ in Eq. (13) 
are those taken at the output face of the crystal. Although the propagation does not change the weight of the modes in the superposition, it might change the relative phases of the complex amplitude.

Finally, the measurement of the Wigner function of the signal photon alone, without taking into account the idler photon, can be done collecting the idler photon with a multimode fiber (MMF) in order to avoid spatial filtering. In this case, the propagation of the idler mode from the crystal to the detector is not relevant, as the idler state is traced out.

In order to properly compare the results obtained with these experimental setups, with our previous theoretical results, an important element should be considered: the effects on the Wigner function of the propagation of the fields from the output face of the nonlinear crystal to the detectors. In the situations described so far, we have shown the Wigner function at the output face of the crystal. Let us now consider a more realistic situation, in which both photons propagate from the output face of the crystal to the interferometer, maybe traversing a lens in their way. As we have already mentioned, the idler propagation can be properly accounted for by using the correct values of $q_{1}$ and $\mu_{x}$. Thus, we are left with only the propagation of the signal. In the case of the remote preparation of the signal photon (Sec. IV), the effect of the propagation is trivial. The overall shape of the Wigner function would still be described by Eqs. (12) and (15), but with different values for the characterizing parameters.

The most interesting case is the one described in Sec. V, where the state of the idler photon is disregarded. If we use a lens of focal length $f$ located a distance $L_{1}$ after the output face of the nonlinear crystal, and located at a distance $L_{2}$ from the Sagnac interferometer $\left(M_{s}\right)$, the state of the signal photon at the output face of the nonlinear crystal is transformed as

$$
\left|\Psi_{s, i}^{\prime}\right\rangle=\hat{U}_{s} \otimes \hat{I}_{i}\left|\Psi_{s, i}\right\rangle
$$

$U_{s}$ is an unitary transformation over the signal photon, given by the product of the transformations due to the free propagation and the effect of the lenses,

$$
\hat{U}_{s}=\hat{U}_{L_{2}} \hat{U}_{f} \hat{U}_{L_{1}}
$$

$\hat{U}_{L_{n}}$ and $\hat{U}_{f}$, in transverse momentum space, have the form

$$
\begin{gathered}
\left\langle q\left|\hat{U}_{L_{n}}\right| q^{\prime}\right\rangle=\exp \left(-i \frac{L_{n}}{2 k} q^{2}\right) \delta\left(q-q^{\prime}\right) n=1,2, \\
\left\langle q\left|\hat{U}_{f}\right| q^{\prime}\right\rangle=\frac{1}{2 \pi} \int d x \exp \left[i\left(q-q^{\prime}\right) x\right] \exp \left(-i \frac{k}{2 f} x^{2}\right) .
\end{gathered}
$$

We take into account this more realistic scenario to calculate the Wigner function of the signal photon alone. Making use of the new $\left|\Psi_{s, i}^{\prime}\right\rangle$ state in Eq. (17), the new expression for the Wigner function is

$$
\begin{aligned}
W_{s}^{\prime}\left(q_{s}, x_{s}\right)= & \frac{1}{\sqrt{\pi}} \sqrt{\frac{H_{R}^{\prime}-G^{\prime}}{H_{I}^{\prime}+G^{\prime}}} \exp \left[-2\left(H_{R}^{\prime}-G^{\prime}\right) q_{s}^{2}\right] \\
& \times \exp \left\{-\frac{\left[x_{s}-2 H_{I}^{\prime} q_{s}\right]^{2}}{2\left(H_{R}^{\prime}+G^{\prime}\right)}\right\},
\end{aligned}
$$

where

$$
\begin{gathered}
G^{\prime}=\left(\frac{f}{2 k}\right)^{2} \frac{\left|G^{1}\right|^{2}}{\left(2 H_{R}^{1}\right)}, \\
H^{\prime}=H_{R}^{\prime}+i H_{I}^{\prime}=\left(\frac{f}{2 k}\right)^{2}\left(\frac{1}{A+B-i T_{1}}+\frac{\left(G^{1}\right)^{2}}{2 H_{R}^{1}}\right)-i T_{2}, \\
G^{1}=G_{R}^{1}+i G_{I}^{1}=\frac{B-A}{A+B-i T_{1}}, \\
H^{1}=H_{R}^{1}+i H_{I}^{1}=A+B-\frac{(B-A)^{2}}{A+B-i T_{1}}, \\
T_{1}=\frac{f-L_{1}}{2 k}, \\
T_{2}=\frac{f-L_{2}}{2 k} .
\end{gathered}
$$

Let us to note that Eqs. (29) and (18) have the same mathematical structure.

In Figs. 4(b) and 4(d) we show two examples of how the consideration of the propagation of the signal photon affects the shape of the Wigner function retrieved. Figures 4(a) and 4(c) show the Wigner function at the output face of the nonlinear crystal, without considering the propagation effects (as described in Sec. V). Figures 4(b) and 4(c) plot similar cases, but now taking into account the propagation from the output face of the nonlinear crystal to the entrance of the Sagnac interferometer, showing clearly how the shape of the Wigner function of the signal photon changes. However, it should be noted that this transformation preserves the purity of the signal photon, as no filtering occurs in the process. This can be mathematically shown by inserting the expressions for $H^{\prime}$ and $G^{\prime}$ in Eq. (21). Note that Fig. 4(d) is exactly the same than Fig. 1(b), although they represent two very different experimental situations. In the case of Fig. 1(b) the signal photon results in a coherent Gaussian state with a plane wave front by means of projecting the idler photon onto a given state. In Fig. 4(d), the signal photon results on exactly the same state but without any postselection.

\section{B. Wigner function of the two-photon state}

The Wigner function of the two-photon state can be calculated using the more general Eq. (3). It can be readily shown that at the output face of the crystal it has the form 


$$
\begin{aligned}
& W_{s, i}\left(q_{s}, x_{s}, q_{i}, x_{i}\right) \\
& =\frac{1}{\pi} \exp \left\{-2 \operatorname{Re}(A)\left(q_{s}-q_{i}\right)^{2}-2 \operatorname{Re}(B)\left(q_{s}+q_{i}\right)^{2}\right\} \\
& \quad \times \exp \left\{\frac{-\left[2 \operatorname{Im}(B-A)\left(q_{i}-q_{s}\right)+x_{s}\right]^{2}}{\operatorname{Re}(A+B)}\right\} \\
& \quad \times \exp \left\{-\frac{\left[2 \operatorname{Im}(B-A)\left(1-\nu_{1}\right)\left(q_{s}-q_{i}\right)+x_{i}-\nu_{1} x_{s}\right]^{2}}{2\left(H_{R}-G\right)}\right\}
\end{aligned}
$$

where

$$
\nu_{1}=\frac{\operatorname{Im}(B-A)}{\operatorname{Re}(A+B)} .
$$

This Wigner function can be measured experimentally using an extension of the procedure to measure the Wigner function of the signal alone. In this case, instead of collecting the idler photon with a multimode fiber, it will pass through another Sagnac interferometer similar to the one used for the signal photon, as it is sketched in 6. Under these experimental conditions, the coincidence rates from the output ports of both Sagnac interferometers will have the following shape:

$$
\begin{aligned}
R_{c} \propto & I_{s}^{1}\left(I_{i}^{1}+I_{i}^{2}\right)+I_{s}^{2}\left(I_{i}^{1}+I_{i}^{2}\right)+\left(I_{s}^{1}+I_{s}^{2}\right) W_{i}\left(x_{i}, q_{i}\right) \\
& +\left(I_{i}^{1}+I_{i}^{2}\right) W_{s}\left(x_{s}, q_{s}\right)+W_{s, i}\left(x_{s}, q_{s}, x_{i}, q_{i}\right)
\end{aligned}
$$

where $I_{j}^{n}$, with $n=1,2$ and $j=s, i$, are the counts corresponding to a signal or idler photon which travels through either path of the interferometer. $W_{j}\left(x_{j}, q_{j}\right)$ is the Wigner function of the signal (idler) photon alone regardless of the state of the idler (signal) photon, as described in Sec. V. $W_{s, i}\left(x_{s}, q_{s}, x_{i}, q_{i}\right)$ is the Wigner function of the two-photon state at the output face of the crystal and can be extracted from the coincidences of the two outputs, after subtracting the background elements.

\section{CONCLUSIONS}

In this paper we have presented some useful results regarding the spatial Wigner function of entangled paired photons. We have studied the remote preparation of one of the photons of the pair, showing explicit results for the photon being in a Gaussian beam and in a superposition of two modes. Also, we have studied the important case of the

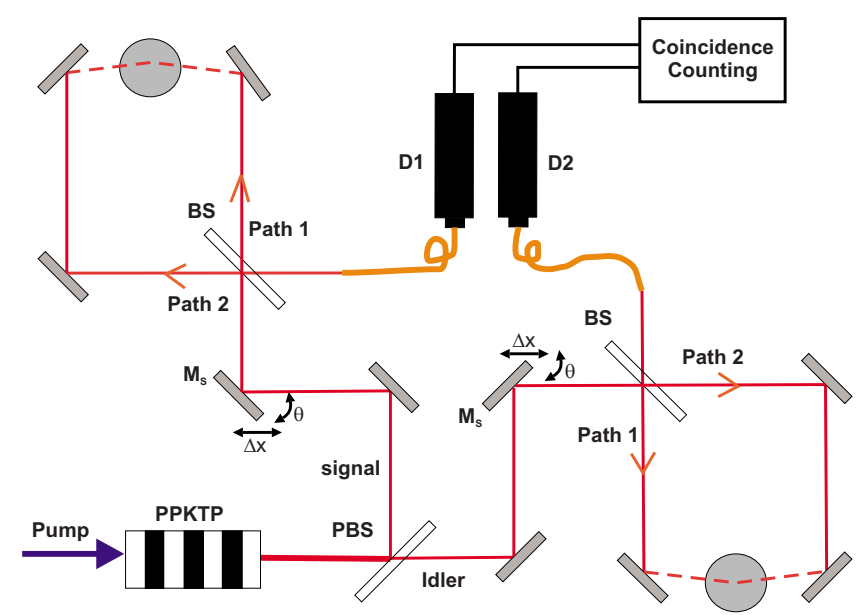

FIG. 6. (Color online) Sketch of the setup for measuring the Wigner function of the spatial state of two photons produced from an SPDC source. In this case, both signal and idler photon are sent to a Sagnac interferometer and detected by Detector 1 and Detector2, respectively, after been collected by a multimode fiber. Using coincident logical detection and after subtracting the coincident background, we obtain the spatial Wigner function corresponding to the two-photon state.

Wigner function of only one of the two photons of the pair, which shows that one photon of the pair is in a statistical mixture of modes. This case can be particularly important to estimate the amount of spatial entanglement of the source. Finally, we propose an experiment to completely measure the spatial state of the two photons, by using a pair of Sagnac interferometers. We have given analytical expressions for the results one would expect in this case.

All these analytical studies show that the spatial degree of freedom of photons can be used to explore the physics of continuous variable systems. Also, it will allow us to further control the spatial state of the photons.

\section{ACKNOWLEDGMENTS}

This Work was supported by the European Commission (Qubit Applications, Contract No. 015848) and by the Government of Spain [Consolider Ingenio 2010 (QOIT) CSD2006-00019 and FIS2007-60179].
[1] A. Ekert and A. Zeilinger, in The Physics of Quantum Information, edited by D. Bouwmeester (Springer, Berlin, 2000).

[2] D. Bouwmeester, J. W. Pan, K. Mattle, M. Eibl, H. Weinfurter, and A. Zeilinger, Nature (London) 390, 575 (1997).

[3] N. Gisin, G. Ribordy, W. Tittel, and H. Zbinden, Rev. Mod. Phys. 74, 145 (2002).

[4] J. C. Howell, R. S. Bennink, S. J. Bentley, and R. W. Boyd, Phys. Rev. Lett. 92, 210403 (2004).

[5] G. Molina-Terriza, J. P. Torres, and L. Torner, Nat. Phys. 3,
305 (2007).

[6] G. Molina-Terriza, J. P. Torres, and L. Torner, Phys. Rev. Lett. 88, 013601 (2001).

[7] G. Molina-Terriza, A. Vaziri, R. Ursin, and A. Zeilinger, Phys. Rev. Lett. 94, 040501 (2005).

[8] S. P. Walborn, P. H. Souto Ribeiro, L. Davidovich, F. Mintert, and A. Buchleitner, Nature (London) 440, 1022 (2006).

[9] A. K. Jha, B. Jack, E. Yao, J. Leach, R. W. Boyd, G. S. Buller, S. M. Barnett, S. Franke-Arnold, and M. J. Padgett, Phys. Rev. 
A 78, 043810 (2008).

[10] A. Royer, Found. Phys. 19, 3 (1989).

[11] A. I. Lvovsky and M. G. Raymer, Rev. Mod. Phys. 81, 299 (2009).

[12] G. Breitenbach, S. Schiller, and J. Mlynek, Nature (London) 387, 471 (1997).

[13] A. I. Lvovsky, H. Hansen, T. Aichele, O. Benson, J. Mlynek, and S. Schiller, Phys. Rev. Lett. 87, 050402 (2001).

[14] G. F. Calvo, Opt. Lett. 30, 1207 (2004).

[15] R. Simon and G. S. Agarwal, Opt. Lett. 25, 1313 (2000).

[16] A. Royer, Phys. Rev. A 15, 449 (1977).

[17] K. Banaszek and K. Wodkiewicz, Phys. Rev. A 58, 4345
(1998).

[18] S. P. Walborn, A. N. de Oliveira, S. Padua, and C. H. Monken, Phys. Rev. Lett. 90, 143601 (2003).

[19] K. W. Chan, J. P. Torres, and J. H. Eberly, Phys. Rev. A 75, 050101(R) (2007).

[20] Clara I. Osorio, G. Molina-Terriza, and J. P. Torres, Phys. Rev. A 77, 015810 (2008).

[21] G. S. Agarwal, Phys. Rev. A 3, 828 (1971).

[22] A. Serafini, F. Illuminati, and S. De Siena, J. Phys. B 37, L21 (2004).

[23] Brian J. Smith, Bryan Killet, M. G. Raymer, I. A. Walmsley, and K. Banaszek, Opt. Lett. 30, 3365 (2005). 